murine strain compared to wild-type mice. Further experiments are necessary to further elucidate the kinetics of $T$ pallidum-infected Myd-88 -/- mice (relative DNA burden in tissue compartments, carrying out infection to 3 and 6 months and beyond to assess chronicity). MyD-88 deficient mice may hold the promise of serving as one of the first useful murine models to study immunopathogenesis of $T$ pallidum infection. Abstract O4-S2.05 figure 1: representative epididymus sections from Day 21 sacrifice. Formalin-fixed tissues were stained with $\mathrm{H} \& \mathrm{E}$ as well as $T$ pallidum-specific immunohistochemical stain (IHC).

\section{4-S2.06 A PRIMATE MODEL OF MYCOPLASMA GENITALIUM CERVICAL INFECTION}

doi:10.1136/sextrans-2011-050109.150

P Totten, G Wood, S Iverson-Cabral, P Cummings, Y Cosgrove Sweeney, D Patton. University of Washington Seattle, USA

Background Mycoplasma genitalium (MG) is a newly recognised pathogen associated with acute and persistent reproductive tract infection in men and women. Understanding of the disease mechanisms, persistence and immune avoidance of this organism is hampered by the lack of a suitable animal model.

Methods Female pigtail macaques (Macaca nemestrina) were inoculated cervically with $\sim 109$ genome equivalents ( $\sim 108$ ccu's) of MG strain G37, then assessed at intervals over 8 weeks for the persistence of MG in lower tract specimens. Fallopian tube biopsies were collected via laparotomy at Weeks 4 and 8. Specimens were assessed for the presence of MG DNA by qPCR and for viable MG by growth in $\mathrm{H}$ broth and Vero cell co-cultures. Serum collected at intervals was evaluated by immunoblot and ELISA for reactivity to $\mathrm{MG}$ antigens. Finally the variable regions of the immunodominant surface antigens, MgpB and MgpC, were analysed by PCR cloning and sequencing to evaluate sequence variation during infection.

Results Of the five primates inoculated cervically with MG, three were infected throughout the 8 weeks of the study, one maintained infection for 4 weeks and one resisted infection. Recovery of viable MG from lower reproductive tract sites was improved by co-culture in Vero cells followed by qPCR to measure an increase in MG genomes during culture. Growth in $\mathrm{H}$ broth, as determined by colour change proved an unreliable indicator of the presence of viable $M G$ in the specimen possibly due to the presence of primate microorganisms that inhibit the growth of MG. No viable $M G$ or MG DNA was detected in upper tract tissues in any of the primates perhaps suggesting that longer infection times or repeated inoculations are needed to achieve ascension in this model. Analysis of mgpB variable regions $B$ and $G$ indicated that after 8 weeks of infection the predominant expressed sequence changed from that of the G37C inoculum to 1 to 5 novel sequences consistent with recombination between the expression site and the MgPars. In contrast, no sequence variation was observed in the inoculum grown in vitro for a similar duration. Antibodies reactive with $\mathrm{MG}$ antigens, including the variable regions of $\mathrm{MgpB}$ and $\mathrm{MgpC}$, were detected by immunoblot and ELISA in serum and cervical exudates.

Conclusions The cervical inoculation model of pigtail macaques results in long-term infection and can be used to study the persistence of MG, development of antibodies and antigenic variation.

\section{Health services and policy oral session 1 -Innovation technology 05-S1.01 EMPLOYING SCHOOL NURSES AS A HEALTHCARE POINT
OF CONTACT FOR MALE HIGH SCHOOL STUDENTS: A
SCHOOL-BASED INTERVENTION TO PREVENT STD, HIV,
AND TEEN PREGNANCY}

doi:10.1136/sextrans-2011-050109.151

${ }^{1} \mathrm{P}$ Dittus, ${ }^{2} \mathrm{C}$ De Rosa, ${ }^{3} \mathrm{R}$ Jeffries, ${ }^{3} \mathrm{~A}$ Afifi, ${ }^{3} \mathrm{~W}$ Cumberland, ${ }^{1} \mathrm{~K}$ Ethier, ${ }^{2} \mathrm{E}$ Chung, ${ }^{4} \mathrm{E}$ Martinez, ${ }^{5} \mathrm{R}$ Loya, ${ }^{4} \mathrm{P}$ Kerndt. ${ }^{1}$ Centers for Disease Control and Prevention Atlanta, USA; ${ }^{2}$ Los Angeles County Department of Public Health, USA; ${ }^{3}$ UCLA, USA; ${ }^{4}$ Health Research Association, USA; ${ }^{5}$ Los Angeles Unified School District, USA

Background Adolescent males tend to have lower levels of knowledge about sexual and reproductive health (SRH), and access health care less frequently than adolescent females. Innovative strategies are needed to reach males with accurate information and resources regarding their SRH needs. Such strategies may improve adolescent males' access to SRH services, including STD screening, treatment, and contraception.

Methods A multi-level intervention was delivered and evaluated across 5 years in a large public school district in Los Angeles, California. One intervention component sought to improve students' awareness and utilisation of condom availability programs (CAPs) in schools by working with key school personnel, particularly nurses, to more effectively implement district CAP policies. Six intervention and six control high schools participated in the study. Analyses included survey data from 13733 high school males across 5 years (T1-T5). A mixed model logistic regression analysis was used to test for an intervention effect on males' reports of services sought from the school nurse. Random effects on the student level were included to control for repeated measures on the same student.

Results The sample was 80\% Latino and 9\% African American; the mean age was 16.3. In the intervention as compared to the control condition, statistically significant increases were observed across 5 years of intervention in respondents' reports of going to the school nurse for information about birth control, STDs, pregnancy, or sex (see Abstract O5-S1.01 figure 1), as well as reports of going to the school nurse for condoms. For example, $5.4 \%$ of intervention school males reported going to the nurse for condoms at T1; reports increased to $9.6 \%$ at T5 for this group, whereas an opposite trend was observed for control school males.

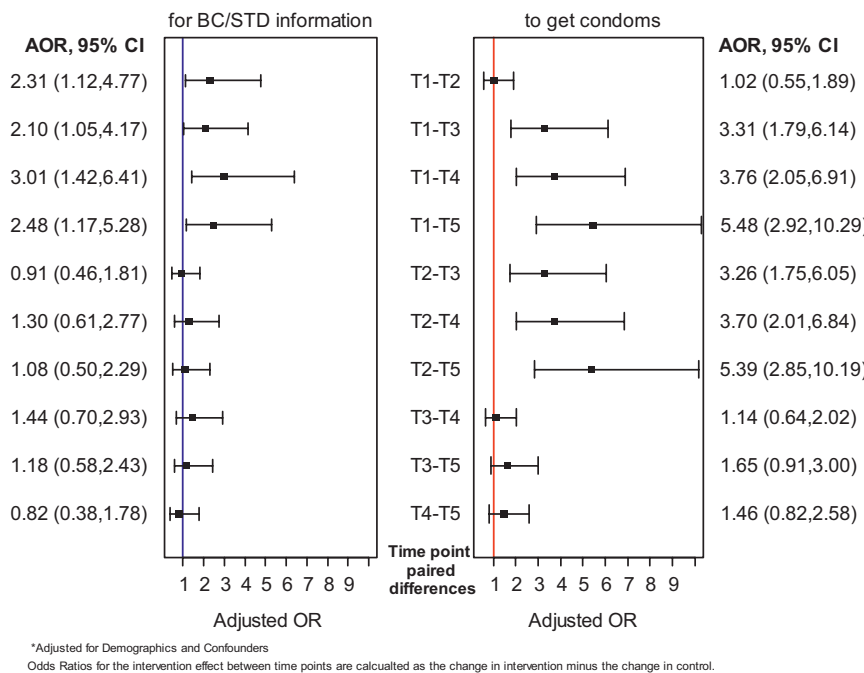

Abstract 05-S1.01 Figure 1 Adjusted ORs* for the change between time points in males going to the school nurse. 